\title{
Mitotically active cellular fibroma of the ovary diagnosis and treatment
} \author{
Sanam Al Houda Brich ${ }^{4}$, Amal Ahmed Al Rashid ${ }^{5}$ and Shereen Amin Elazzazy 1,* \\ ${ }^{1}$ Department of Pharmacy, National Center of Cancer Care and Research, Doha, Qatar. \\ ${ }^{2}$ Department of Medical Oncology, National Center of Cancer Care and Research, Doha, Qatar. \\ ${ }^{3}$ Department of Internal Medicine, Hamad Medical corporation, Doha, Qatar. \\ ${ }^{4}$ Department of obstetrics and gynecology, Woman Recreation Center, Doha, Qatar. \\ 5 Department of Clinical Imaging, Hamad Medical corporation, Doha, Qatar.
}

Rawan Ahmad Dawoud 1, Hind Hamad El-Malik 2, Haajra Fatima Fatima 3, Mohammed Elamin Faris Faris ${ }^{3}$,

GSC Biological and Pharmaceutical Sciences, 2021, 17(02), 160-166

Publication history: Received on 11 October 2021; revised on 20 November 2021; accepted on 22 November 2021

Article DOI: https://doi.org/10.30574/gscbps.2021.17.2.0335

\begin{abstract}
Mitotically active cellular fibroma (MACF) of the ovary is a relatively newly recognized histological disease entity which explains its scarce reporting in literature and unknown prognosis [1]. In this tumor type, high mitotic activity is noticed without severe atypia. These tumors are categorized as benign ovarian tumors with late recurrence, if any. However, we herein report an unusual case of an ovarian mitotically active atypical fibrous tumor case which presented as recurrence within six months of being operated thereby raising suspicion of an element of malignancy. On presentation, the patient suffered from abdominal pain and abdomino-pelvic mass. After imaging confirmed an ovarian neoplastic lesion patient underwent bilateral salpingo-oophorectomy and hysterectomy. Histopathology defined the lesion as "ovarian mitotically active atypical fibrous tumor".
\end{abstract}

Six months later, abdominal pain recurred for which imagining was done and confirmed a newly developed well defined irregular complex solid cystic mass causing intestinal obstruction giving an impression of a malignant neoplasm, probably ovarian carcinoma. However, histopathology for the new lesion remained the same as was for the previous mass, i.e. mitotically active ovarian fibroma, but with lung and mesenteric lymph nodes metastases. Patient couldn't be operated on due to her co-morbid conditions, and the mass was vascular. She was given supportive care with consideration to start hormonal therapy and during this period died due to sepsis.

This case highlights the importance of having a high suspicion of the possibility of malignancy when mitotically active cellular fibroma manifests as a recurrence of mass as this tumor may tend to have an uncertain malignant potential. Thereby, stressing the importance of long term follow up even after the primary tumor is surgically treated without any surgical difficulty or tumor rupture [2]. Such tumors need to be followed and reported more often to help understanding the way they behave and their management.

Keywords: Fibroma; Ovarian tumors; Fibrosarcoma; Ovarian neoplasm

\section{Background}

Mitotically active cellular fibromas (MACF) are rare type of benign tumor with high cellularity rate, that can differ from fibrosarcoma or cellular fibroma if $\geq 4$ mitoses per 10 high-power fields (HPF) without nuclear atypia [3]. The incidence of fibrosarcoma is $\sim 10 \%$ of all fibromas diagnosed cases [4]. This type of aggressive disorder can't be cured and usually characterized with high serum tumor marker CA-125, that is usually treated with surgical interventions. However, $11 \%$

\footnotetext{
${ }^{*}$ Corresponding author: Dr. Shereen Elazzazy, PharmD , MBA, NLP PgDip, Bsc pharm; E-mail:Shereen_amin@yahoo.com Department of Pharmacy, National Center of Cancer Care and Research, Doha, Qatar. 
of the cases that have been diagnosed with cellular fibromas and $13 \%$ of the mitotically active fibromas are metastasize to extraovarian sites at the time of surgery, so these patients need clinical follow up as benign cases [5].

Few cases have been reported with same diagnosis, but the exact pathophysiology of the disease still not well known, clinical guideline for the treatment pathways is needed as the patients usually have good prognosis [6]. Therefore, our reported case is one of these rare cases of MACF of the ovary that had presented with severe symptoms and aggressive nature of the disease.

\section{Case presentation}

A 65-year-old Bahraini lady with multiple comorbidities: diabetes and hypertension, presented for the first time to Hamad Medical Corporation (the main tertiary healthcare body in Qatar) in October 2018, and visited the Gyn oncology clinic for the evaluation of suspicious ovarian mass investigated in Bahrain following the complaints of abdominal distension of 4 months (CA125:80). At this time, the ultrasound showed large pelviabdominal mass likely representing ovarian epithelial neoplasm. She had recurrent attacks of abdominal pain, shortness of breath and vomiting reflecting compression symptoms. Then, had staging imaging (figure 1,2,3) with suspected metastatic lesions in breast and chest.

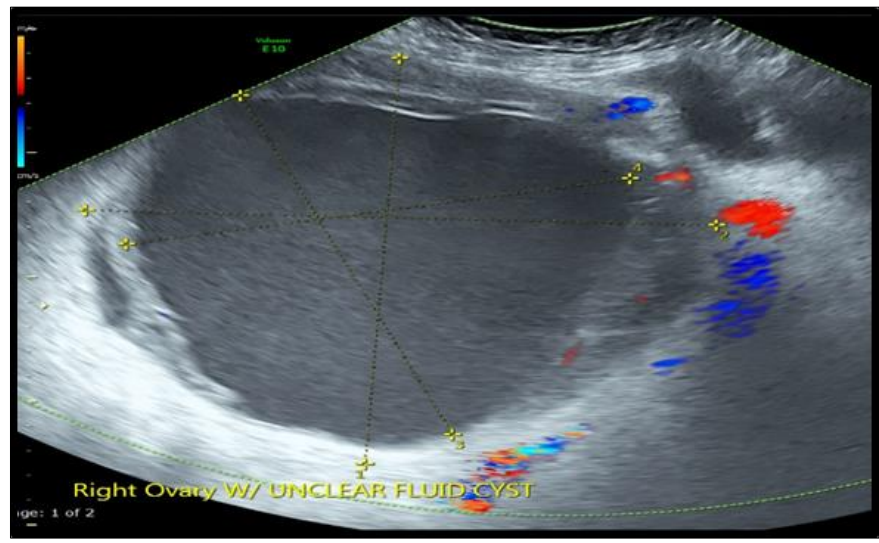

Figure 1 Ultrasound pelvis shows Right adnexal predominantly cystic lesion with peripheral irregular thickened wall and solid components that protruding into the cyst cavity, raising possibility for malignant neoplasm

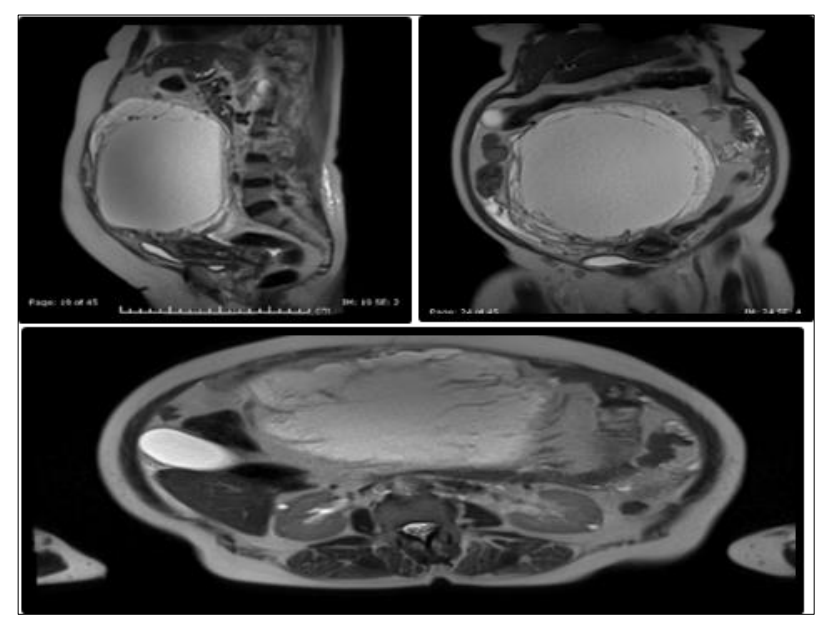

Figure 2 Limited MRI Pelvis (T2WI a. sagittal, b. coronal c. axial planes) shows right multilocular pelviabdominal cystic mass with peripheral incomplete septae and thickened wall(arrow) 

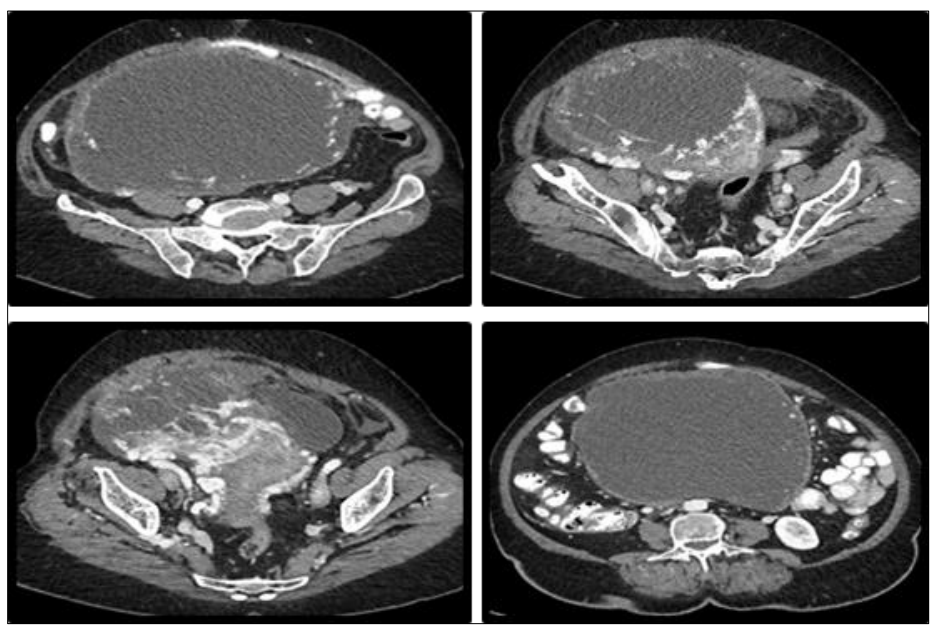

Figure 3 Contrast-enhanced CT scan (staging protocol) shows a large cystic mass with multiple peripheral enhanced septa and calcifications mainly at its caudal end. Imaging findings suggest a right ovarian neoplastic lesion

Following, a sudden deterioration in her symptoms, a decision was made to proceed for surgery that do not involve full surgical staging as patient was not deemed fit for lengthy procedure under anesthesia. Laparotomy - TAH + BSO + appendicectomy + small bowel excision and re-anastomosis done in November 2018.

Intraoperatively, there was a moderate volume of ascites, no omental or upper abdominal disease, a large complex mass was arising from right ovary/right uterine cornua. Moreover, the patient found to have bowel densely adherent superiorly and posteriorly.

Local pathological examination of the ovarian cyst measured $18 \times 16 \times 7.5 \mathrm{~cm}$, and had thick irregular walls covered with adhesions and hemorrhages. And the right ovarian tumor is partially cystic partially solid with hemorrhagic changes and coagulative necrosis. The tumor has fibrous looking cells showing mild to moderate nuclear atypia and very high mitosis (up to 15/ 10 high-power fields) and coagulative necrosis. The endomyometrium was atrophic with cystic changes. Where the myometrium showed adenomyosis, the Cervix had multiple nabothian cysts. But the Left fallopian tube and left ovary appeared without significant pathology.

Furthermore, extensive bone formation was noted, associated with occasional atypical looking cells similar to the background tumor. Parts of the small intestine appeared with focal adhesions with no evidence of tumor infiltrate. However, ischemic necrosis and features of perforation were noted, but peritoneal fluid was negative for malignancy.

The immunohistochemical stains showed that the tumor was positive for vimentin, CD10, and CD99. There was up to $50 \%$ labeling index by Ki-67. The desmin showed focal nonspecific positivity. But the tumor cells were negative for cytokeratin AE1/3, EMA, progesterone receptor, estrogen receptor, SMA, MNF 116, CK 7, and S100.So, the final pathology result was ovarian mitotically active atypical fibrous tumor, with coagulative necrosis, where fibrosarcoma cannot be excluded.

Pathology slides were reviewed with a second opinion from Mayo clinic, USA which concluded that the lesion is most likely to represent a mitotically active fibroma.

Described the ovarian cyst as sex cord-stromal spindle cell neoplasm with uniformly mild to focally moderate cytologic atypia, brisk mitotic activity (up to 17 figures per $10 \mathrm{HPF}$ ), and large zones of ischemic and hemorrhagic necrosis. Regions of the tumor exhibited metaplastic bone production and scattered limited clusters of intermixed luteinized cells.

Special staining highlighted reticulin fibers around individual tumor cells. Immunohistochemical stains showed patchy weak expression for SF1 in fibrous component; which is a sensitive and specific marker for sex cord-stromal tumors of the ovary), and strong expression of calretinin and inhibin in small aggregates of luteinized cells ,both were negative in fibromatous component, and concluded that, since the cells maintained a spectrum of mild to moderate atypia rather than moderate to severe, the features were most in keeping with mitotically active fibroma with osseous metaplasia.

Fibromasarcoma was not the final diagnosis from our lab as the appearance did not meet the criteria for this diagnosis. 
Due its mitotic activity and the possibility of local recurrence, patient was planned for follow-up with the gyn oncology team clinic.
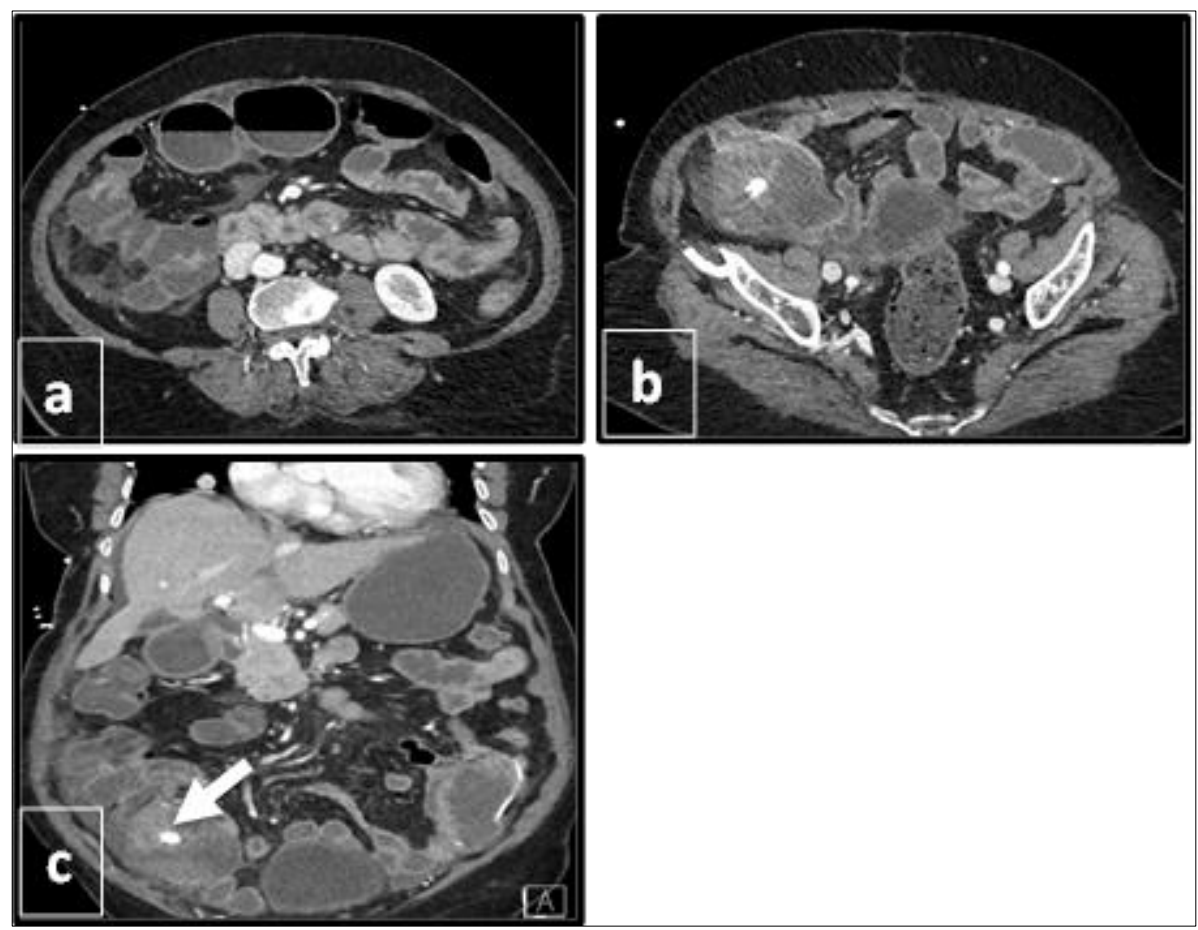

Figure 4 CT enterograhaphy image showed (a) mural thickening and mucosal hyperenhancement of the jejunal loops. (b, c) newly developed irregular mixed solid cystic mass with central calcification at the right iliac fossa (arrow), raising possibility of tumor recurrence
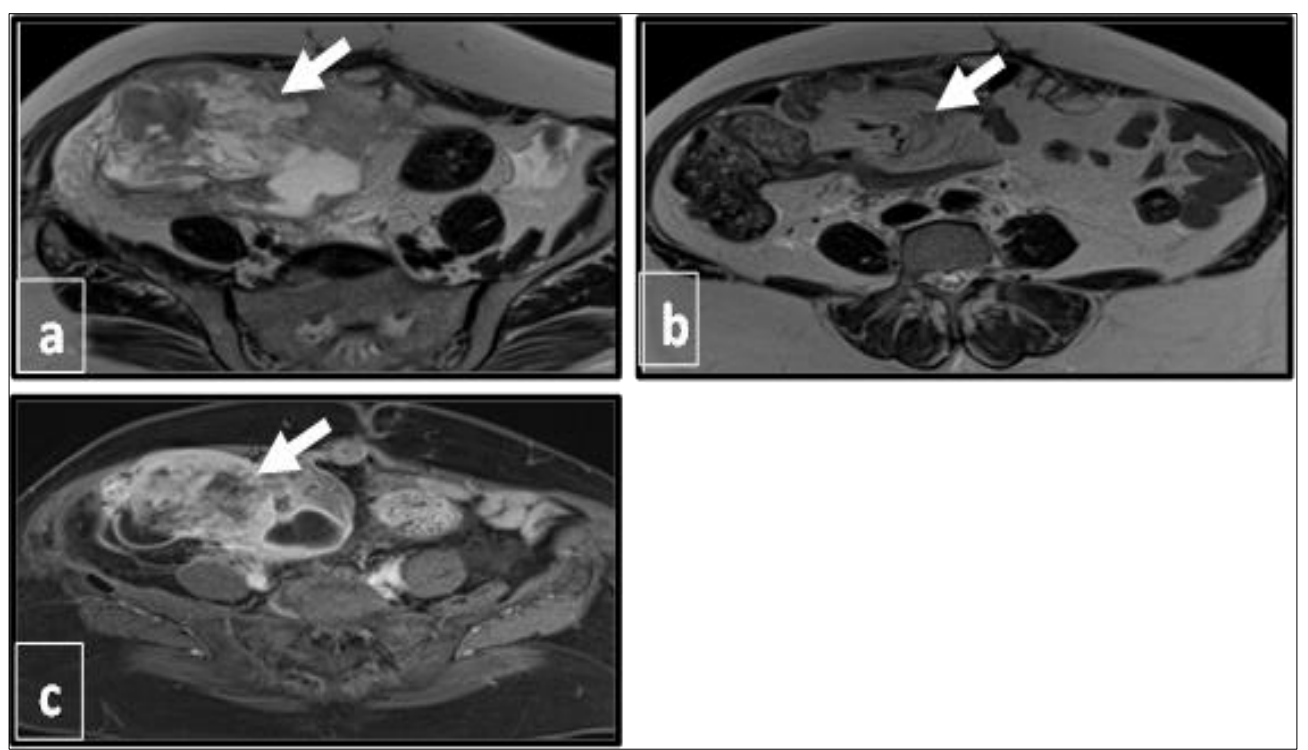

Figure 5 MRI PELVIS (a ,b)Axial T2WI-SE, (a) multilocular mass with irregular papillary projections and solid components; (b) Soft tissue edema evident by fat stranding (arrow) of the right side of the pelvis, however the surrounding bowel loops are clear; (c) Axial T1WI-FS POST IV contrast injection shows shows marked enhancement of the papillary projections and solid components (arrow)

In May 2019, during a surveillance follow up; the patient presented with acute complaints of rectal bleeding and hemodynamic instability. Investigated with conclusion of recurrence at the right iliac fossa (figure 4), a mass lesion CT 
guided biopsy taken, showed similar morphologic appearances, including an osseous component with atypical intervening spindle cells. The immunohistochemical stains revealed that the spindle cells are strongly positive with vimentin and SATB2 but focally positive CK AE1/3 with CD99, BCL-2, CD 56 and TLE. While staining with EMA, MDM2, S100, SOX10, SMA, DESMIN, CALDESMON, STAT6, CD117, CD34, inhibin, calretinin and Beta Catenin are negative. However, the initial biopsy showed abundant osteoid matrix in association with highly mitotically active and atypical cells of osteoblastic origin (highlighted by SATB2), which raised the possibility of heterogenic osteosarcomatous component. A second opinion from Mayo clinic, USA for the recurrent tissue biopsy was reported as mitotically active fibroma with osseous metaplasia. However, ovarian fibrosarcoma couldn't be excluded.

The gyn-oncology surgeon opinion was to go for exploration and resection of the mass with bowel resection and possible colostomy because MRI showed mass lesion that couldn't be separated from one bowel loop in all images (figure 5).

Further imaging (figure 6) revealed same malignant neoplasm of the right lower abdominal quadrant causing small intestinal obstruction, lung metastases and metastatic mesenteric lymph nodes.

Discussed in MDT and decision concluded to defer surgery due to her significant multiple comorbidities, in addition to the extremely vascular nature of the mass that led to massive bleeding in last surgery. Furthermore, embolization by interventional radiology was explored and resolved not fit as well.

Palliative treatment of tamoxifen 20 mg once daily and symptom relieve was given, and patient died on 15 of July 2019.

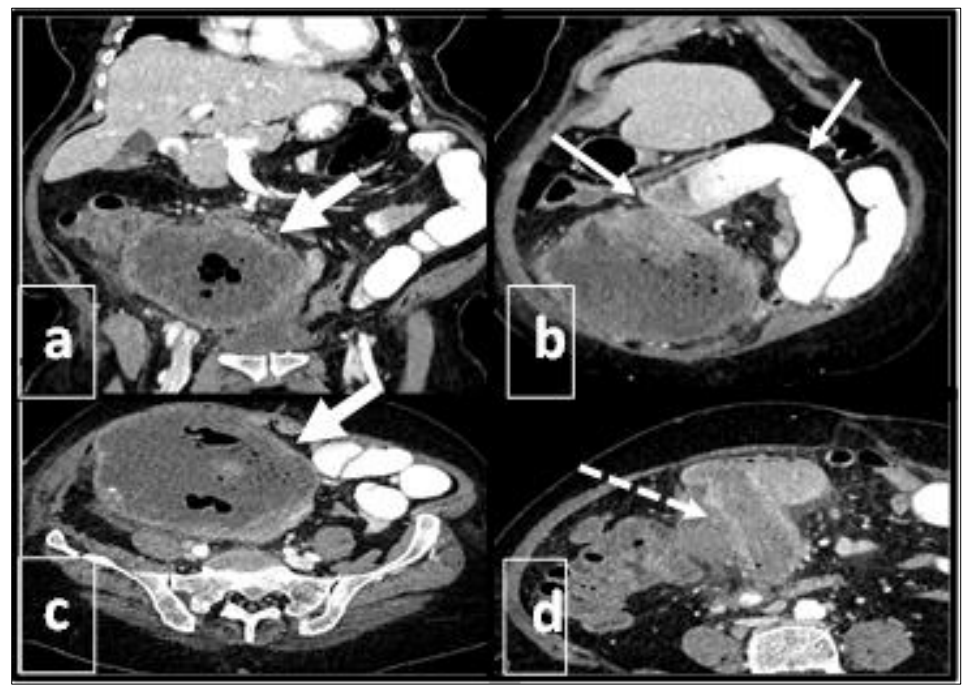

Figure 6 Contrast-enhanced CT scan shows (a , c)Solid lesion (arrow) with central breaking down, gas locules and tiny peripheral calcification located at the right lower abdominal quadrant causing $(b, d)$ interruption and dilatation of the small intestine (thin arrow) probably due to small intestinal invasion (dotted arrow)

\section{Discussion}

According to WHO classification sex cord- stromal Ovarian tumors accounts for $10 \%$ of all the ovarian tumors [7]. Mitotically Active Cellular Fibromas (MACF), fibromas, or fibro sarcomas are subdivisions from the Fibrous ovarian tumors [8]. Over the past years the definition of the mitotic activity was changed since it was first set in 1981 by Pratt and Scully, where there was no difference in mitotic activity in relation to the nuclear atypia to determine the aggressiveness of the fibromatous neoplasm [7,8].

Our patient presented with large complex ovarian lesion as most of the reported cases presented with symptomatic ovarian or pelvic lesions treated surgically. Upon relapse which was 6 months afterwards she was treated conservatively as she wasn't candidate for neither surgical nor radiotherapy. Compared to other reported cases - with regards to the clinical features of the second relapse which was high CA125, extraovarian involvement, $30 \%$ of Ki 67 and positive PR while it was negative previously, and positive NF1; the last one was not mentioned before regarding the clinical relevancy in similar cases. 
On the other hand, the microscopic characteristics of the first pathology were not as most reported cases with more than or equal 4 mitosis per $10 \mathrm{HPF}$; to be irregular, hemorrhagic, and necrotic, which magnify the uniqueness of our case. Mentioning that it had 15 mitosis in $10 \mathrm{HPF}$ which considered being rare in such same cases.

Cytogenetic study, and molecular sequencing is an advanced diagnostic tool under investigation for this rare disease.

The average follow up period was almost 5 years (from 3 months up to 12 years for most of the reported cases, with no evidence of disease recurrence, while other reported one case of disease relapse within 6 years as she was treated with tamoxifen as well, but in our case the relapse was earlier and more aggressive which results in patient's death $[1,5]$.

Treatment modality was the same compared to other reported cases, surgically upon first incidence of disease, but hormonal treatment at the second relapse distinct our case from others as only few of them had the same modality of treatment [9], while the exact approach still not conclusive. Nevertheless, the neoplasm was aggressive and resulted in death which was not reported of such disease known to have long survival and good prognosis.

\section{Conclusion}

In conclusion, mitotic fibromas of the ovary diagnosis from fibro sarcoma, has been changed over years. Genomic sequencing and cytogenetic study are promising diagnostic tool, that need to be verified and clinically validated for the clinical implementation, decision making for such cases, and to differentiate between the MACF and sarcomas, which might impact the treatment pathway and improve survival of these patient. Because of the rareness of the disease, exact pathological and treatment algorithm will be challenging.

\section{Compliance with ethical standards}

\section{Disclosure of conflict of interest}

No conflicts of interest to disclose.

\section{Statement of informed consent}

This paper received waiver of informed consent by Hamad Medical corporation IRB-MRC.

\section{Authors contribution}

Rawan Ahmad Dawoud (R.D.), Hind Hamad El-Malik (H.M.), Haajra Fatima Fatima (H.F.) , Mohammed Elamin Faris Faris (M.F.) , Sanam Al Houda Brich (S.H.) , Amal Ahmed Al Rashid (A.R.) , Shereen Amin Elazzazy (S.E.).

R.D. conceived the presented idea, did the major contribution in writing the manuscript and provided the final version for revision. H.M. reviewed the final version, H.F., M.F. and S.H involved in manuscript writing, A.R. provided the radiographical data and co-write the paper, S.E. supervised the project and contributed with writing the manuscript, the revision of the article and being the corresponding author. All authors read and approved the final manuscript.

\section{References}

[1] Yamada, T., Hattori, K., Satomi, H., Hirose, Y., Nakai, G., Daimon, A., Hayashi, A., Terai, Y., Ohmichi, M., \& Fukunaga, M. (2015). Mitotically active cellular fibroma of the ovary: a case report and literature review. Journal of ovarian research, 8, 65 .

[2] Wu H, Xie J, Huang W, Wu J. (2014). Eur J Gynaecol Oncol, Mitotically active cellular fibroma of the ovary: a case report and a review of the literature, 35(1) , 81-3.

[3] Yıldırım N, Saatli B, Akalın F, Ulukuş Ç, Obuz F, Saygılı U. (2015). Mitotically active cellular fibroma of the ovary: A case report. Turk J Obstet Gynecol , 12(1) , 53-55.

[4] Prat, J., \& Scully, R. E. (1981). Cellular fibromas and fibrosarcomas of the ovary: a comparative clinicopathologic analysis of seventeen cases. Cancer, 47(11), 2663-2670.

[5] Irving, J. A., Alkushi, A., Young, R. H., \& Clement, P. B. (2006). Cellular fibromas of the ovary: a study of 75 cases including 40 mitotically active tumors emphasizing their distinction from fibrosarcoma. The American journal of surgical pathology, 30(8), 929-938. 
[6] Katsuya Matsuda, Seiko Tateishi, Yuko Akazawa, Matsuda et al. (2016). Rapid growth of mitotically active cellular fibroma of the ovary: a case report and review of the literature, Diagnostic Pathology, 11, 101.

[7] Peres, L. C., Cushing-Haugen, K. L., Anglesio, M., Wicklund, K., Bentley, R., Berchuck, A., Kelemen, L. E., Nazeran, T. M., Gilks, C. B., Harris, H. R., Huntsman, D. G., Schildkraut, J. M., Rossing, M. A., Köbel, M., \& Doherty, J. A. (2018). Histotype classification of ovarian carcinoma: A comparison of approaches. Gynecologic oncology, 151(1), 5360.

[8] Grenier, P.A., Agrawal, A., \& Chibbar, R. (2017). A case report of primary ovarian fibrosarcoma.

[9] Bucella, D., Limbosch, J. F., Buxant, F., Simon, P., Fayt, I., Anaf, V., \& Noël, J. C. (2009). Recurrence of mitotically active cellular fibroma of the ovary. Obstetrics and gynecology international, 2009, 803062. 